7th International Workshop on Astronomy and

Relativistic Astrophysics (IWARA 2016)

International Journal of Modern Physics: Conference Series

Vol. 45 (2017) 1760022 (7 pages)

(C) The Author(s)

DOI: $10.1142 / \mathrm{S} 2010194517600229$

\title{
Dormant Black Holes
}

\author{
José Antonio de Freitas Pacheco \\ University of Nice-Sophia Antipolis, Observatoire de la Côte d'Azur \\ F-60304 - Nice Cedex 4 - France \\ pacheco@oca.eu
}

Published 15 August 2017

\begin{abstract}
The growth of supermassive black holes is intermittent, having periods of low accretion when no nuclear activity is seen in the center of the host galaxy. In such dormant state black holes may tidally disrupt stars scattered from the bulge to inside their influence sphere. The resulting debris are partially captured by the black hole forming a short-lived accretion disk, which produces a variable emission dubbed "tidal flare". Some galaxies candidate to have hosted these tidal events are here considered.
\end{abstract}

Keywords: Supermassive Black Holes, Tidal Flares, Accretion Disks.

PACS numbers: 98.70

\section{Introduction}

The detection by the LIGO laser interferometer of the first gravitational wave signal represents one of the strongest evidence in favor of the existence of black holes in the universe. ${ }^{1}$ The signal, dubbed GW150914, is the consequence of the merger of two black holes having masses respectively equal to $36.3 \pm 5.0 M_{\odot}$ and $28.6 \pm$ $4.0 M_{\odot}$. These masses are substantially higher than values estimated for black hole candidates present in galactic X-ray binaries whose average mass is around $8 \pm 3 M_{\odot}$ (see, for instance, Ref. ${ }^{2}$ ). Clearly, very massive progenitor stars are required to form the black holes implied in the GW150914 event. Massive stars can be formed in the early phases of the universe, when the interstellar gas present in proto-galaxies are still metal-poor. In the scenario described in Ref., ${ }^{3}$ a binary system composed of stars having masses respectively equal to $96.2 M_{\odot}$ and $60.2 M_{\odot}$ was formed around $z \sim 3.5$ in a low metallicity (about $3 \%$ of the solar value) medium. After $5.4 \mathrm{Myr}$, the binary system is now constituted by two black holes, the end product of the evolution of those massive stars. In the following 10.3 Gyr, the black holes lose

This is an Open Access article published by World Scientific Publishing Company. It is distributed under the terms of the Creative Commons Attribution 4.0 (CC-BY) License. Further distribution of this work is permitted, provided the original work is properly cited. 


\section{J. A. de Freitas Pacheco}

orbital energy and angular momentum by emitting gravitational waves, leading to a continuous shortening of their separation until the final merging.

On the other side, the presence of supermassive black holes (SMBHs) in the center either of elliptical galaxies or bulges of spiral galaxies seems presently to be well established and the most dramatic case is our own galaxy. Adaptive optics and speckle imaging with the Keck telescope permitted to derive the orbital parameters of several stars near the galactic center and, in particular, of the star $S 0-16$ that has a pericenter of about $2.9 \times 10^{-4} \mathrm{pc}$ (see, for instance, Ref. ${ }^{4}$ ). The inferred mass of central object is $4.1 \times 10^{6} M_{\odot}$ according to Ref. ${ }^{5}$ This implies a minimum mass density for the object driving the orbital motion of $S 0-16$ of at least $4 \times 10^{16} M_{\odot} / p c^{3}$. Excepting a black hole, no other (black) astrophysical object having such a high density is presently known.

There are different scenarios proposed in the literature trying to explain the formation of these SMBHs but the growth of "seeds" by accretion inside the nucleus of the host galaxy, including also some contribution from coalescences during merger events, is the road that will be privileged here. The "seeds" are probably black holes with masses in the range $100-500 M_{\odot}$, originated from the evolution of massive stars of first generation. These stars would have been formed around $z \sim 15-20$ in the high density peaks of the primordial fluctuation spectrum ${ }^{6}$ and their high masses could have been the consequence of a very inefficient gas cooling at zero metallicity. Besides forming $\mathrm{BH}$ seeds, these massive stars could also have contributed to the process of reionization of the universe. The continuous growth of black holes is consistent with the fact that the present cosmological BH mass density agrees with the accretion (baryonic) mass density derived from the bolometric luminosity function of quasars ${ }^{7-8}$. The dark matter contribution to the growth process is small, never surpassing $10 \%$ of the total amount of the accreted material. ${ }^{9}$

Cosmological simulations following both the formation and the evolution of galaxies and black holes suggest that the growth of the later is intermittent, namely, BHs grow during phases of important accretion when they drive the nuclear activity of the galaxy. ${ }^{10}$ This active phase is generally followed by a "dormant" or quiescent state (like the present case of our Galaxy), when the accretion rate is very low. A new period of growth re-starts as soon as the galaxy captures gas either through the filaments that constitute the cosmic web or when it participates of a merger event.

In this paper, it will be shown that even in a dormant state, the presence of a SMBH in the center of the host galaxy can be inferred if a star is scattered by collisions and penetrate into the influence sphere of the black hole. In this case, as it will be discussed below, the star may be tidally disrupted and the debris is responsible for a luminous flare with timescales of the order of few years. Detailed modeling of these tidal flares provides information about the black hole itself and the nearby stellar population. 


\section{Tidally Disrupted Stars}

Stars in the central region of a galaxy can be scattered via close dynamical encounters and be placed into an orbit inside the influence sphere of the central SMBH. If the tidal forces generated by the black hole overcomes the self-gravity of the star, a disruption event may occur. A fundamental condition for a star be disrupted is that the tidal radius be outside of the black hole horizon. This implies, for a typical main-sequence star of $0.25 M_{\odot}$, an upper limit of about $\sim 10^{8} M_{\odot}$ for the black hole mass, otherwise the star will be directly swallowed through the horizon. Such a mass limit is more stringent if the star is a white-dwarf or a neutron star, since in these cases the upper limit for the black hole mass is respectively $6 \times 10^{5} M_{\odot}$ and $17 M_{\odot}$.

The physics of the disruption process depends essentially on two dimensionless parameters: the first one $(\lambda)$ is defined by the ratio between the tidal radius $r_{T}$ and the pericenter distance $r_{p}$, while the second one $\left(\eta^{2}\right)$ is defined by the ratio between the self-gravity of the star and the tidal-gravity at the surface, when the star is at pericenter. The first parameter essentially defines a condition for disruption based on the specific orbital angular momentum $J$ of the star, which must be less than the critical value

$$
J_{c r i t}=\sqrt{G M_{b h} r_{T}} .
$$

A recent numerical investigation ${ }^{11}$ has shown that if $\lambda \geq 1.8$, the star is totally disrupted and about a half of its mass remains bound to the BH. For $\lambda<1.8$ the star is only partially stripped of its outer envelope and if $\lambda<0.5$ no mass loss occurs.

Most of the investigations on the evolution of the tidal debris assume that the rate at which the bound material returns to the pericenter and circularizes, forming an accretion disk, is identical to the rate at which the black hole accretes the debris material. In fact, this is not true since there is a time delay between the beginning of the circularization process and the instant at which the accretion by the black hole initiates. ${ }^{12}$ Once the circularization process begins, two distinct phases can be distinguished. An early and short phase in which the $\mathrm{BH}$ is not accreting the debris since the gas has not yet reached the last stable orbit. This time interval between the beginning of the circularization and the beginning of the accretion process is fixed by the viscous timescale $t_{a} \sim r^{2} / \nu$, where $\nu$ is the kinematic viscosity that controls the mass flux through the disk. In general, the level of turbulence controls the viscosity and the inward mass flux through the disk. In this phase the flare begins to develop and its luminosity raises very rapidly. The second phase begins once the accretion process by the $\mathrm{BH}$ initiates and it coincides approximately with the instant of maximum flare luminosity.

Several tidal flares candidates have been detected in the optical ${ }^{13-15}$ and $\mathrm{UV}^{16-18}$ spectral bands. However, tidal disruption events are more dramatic seen in the X-ray band since the contrast between the quiescent emission of the host galaxy and that of the tidal disk can attain factors as large as 1000. One example is the 


\section{J. A. de Freitas Pacheco}

$S 0$ galaxy NGC $3599(z=0.0027)$ in which the satellite XMM-Newton detected in 2003 an X-ray emission about two orders of magnitude higher than the upper limit derived by ROSAT in 1993. The evolution of the X-ray emission was modeled in Ref. ${ }^{12}$ where the authors, using non-steady hydrodynamic models for the accretion disk, were able to explain the flare evolution by considering the disruption of a one solar mass star by a $10^{7} M_{\odot}$ black hole. However, this interpretation was recently questioned in Ref. ${ }^{19}$ since the authors claim that NGC 3599 was already bright 18 months before the detection by XMM-Newton. In fact, the X-ray curve derived in Ref. ${ }^{12}$ predicts that the maximum emission occurred about 1.5 years before the XMM-Newton detection in 2003, being consistent with the reported X-ray flux by Ref., ${ }^{19}$ since in this case such an emission would correspond to the fast raising side of the X-ray flare curve. Therefore, these recovered data are not inconsistent at all with the model proposed in Ref. ${ }^{12}$

Two other candidates can be mentioned: the first is XMMSL1 (or SDSS J132342.3+482701) that was detected by XMM-Newton in 2003. The optical counterpart is a galaxy at $z=0.0875$, having a central velocity dispersion of $80 \pm 10$ $\mathrm{km} / \mathrm{s}$. Using this value and the $M_{b h}-\sigma$ relation, the mass of the central SMBH is estimated to be around $2 \times 10^{6} M_{\odot}$. The X-ray light curve modeled in Ref. ${ }^{12}$ requires that the mass of disrupted star be about $1.0 M_{\odot}$ and that mass of the $\mathrm{SMBH}$ be approximately $3 \times 10^{6} M_{\odot}$, consistent with the previous independent estimate. The second candidate is an event associated to a galaxy (SDSS J131122.15-012345.6) located in the cluster Abell $1689(z=0.183)$. No X-ray emission was seen either by the space observatory Chandra in 2000 or by XMM-Newton in 2001. However, in 2004 a variable X-ray emission was detected by Chandra. Since there are few data points in the X-ray curve, the parameters of the model are not well constrained but the existing observations can be explained by tidal flare model in which a star of $0.5 M_{\odot}$ is disrupted by a $10^{7} M_{\odot} \mathrm{SMBH}^{12}$

A controversial candidate is the LINER galaxy IC $3599(z=0.0212)$. A variable X-ray emission was detected by ROSAT in 1991, decaying by two orders of magnitude in a timescale of about one year. ROSAT observations were interpreted as a tidal event in Ref. ${ }^{12}$ and modeled by the disruption of a one solar mass star by a $10^{7} M_{\odot}$ SMBH. However, according to further observations by Chandra in 2002 and XRT-Swift in 2010, the source recovered a state of high X-ray emission. ${ }^{20}$ It is difficult to interpret this new nuclear activity as a second tidal episode since the expected rate for these events is about one per galaxy every $10^{5 \pm 1}$ years. An alternative scenario is that of a star in a highly eccentric orbit that is only partially stripped of its outer envelope during the pericenter passage, occasion at which a tidal flare is produced. ${ }^{20}$ Thus, as mentioned previously, partial disruption occurs if $\lambda<1.8$. Using explicit expressions for the tidal radius and the perigee distance, one obtains

$$
\lambda=0.0115(1-e)^{-1} \frac{m_{*}^{0.31}}{P^{2 / 3}} .
$$


In this equation the mass of the star $m_{*}$ is given in solar units and the orbital period $P$ is given in years. The numerical coefficient in eq. (1) was obtained under the assumption that the mass distribution of the disrupted star can described by the Roche model. The radius of the star was eliminated by using a mass-radius relation valid for Main Sequence (MS) stars. Notice that the expression above does not depend on the black hole mass. On the other hand, from the hydrodynamic models computed in Ref., ${ }^{12}$ it is possible to derive an expression for the X-luminosity (in the band $0.2-2.0 \mathrm{keV}$ ) at maximum, which is given by

$$
L_{x}=6.7 \times 10^{47} \frac{f_{*}}{\mathcal{R}} m_{*}^{0.54} \mathrm{erg} / \mathrm{s} .
$$

In the equation above $f_{*}$ is the mass fraction lost by the star during the pericenter passage, $\mathcal{R}$ is the critical Reynolds number controlling the turbulence level in the disk and $m_{*}$ is the original mass of the star in solar units. Here, it was used again the mass-radius relation for MS stars. In order to illustrate this scenario, let us assume that the stripped star has a mass of about $3.2 M_{\odot}$, corresponding to an A0V star. Since at maximum the X-ray luminosity is about $8 \times 10^{43} \mathrm{erg} / \mathrm{s}$ and considering also a critical Reynolds number of $\mathcal{R}=1000$, one obtains from eq.(2) that the mass fraction lost by the star is $f_{*}=0.064$, a value consistent with numerical experiments. Moreover, the peak X-ray luminosity can be also writen as a function of the accretion rate at maximum, namely

$$
L_{x}=\varepsilon \frac{d M_{p}}{d t} c^{2},
$$

where $\varepsilon \sim 0.09$ is the radiative efficiency of the accretion process (see, for instance, Ref. $^{21}$ ). Thus, from eq.(3), the accretion rate at maximum should be around $d M_{p} / d t$ $=0.016 M_{\odot} / y r$. On the other hand, using the fit resulting from simulated data provided by Ref. ${ }^{11}$ and the mass-radius relation for MS stars, the accretion rate at maximum can be given by the empirical relation

$$
\frac{d M_{p}}{d t}=0.962 A(\lambda) m_{*}^{1.04}\left(\frac{10^{6} M_{\odot}}{M_{b h}}\right)^{1 / 2} M_{\odot} / y r .
$$

In the equation above $M_{b h}$ is the black hole mass in solar units and $A(\lambda)$ is a function of the parameter $\lambda$ derived numerically from the aforementioned simulations. ${ }^{11}$ From eq.(4), assuming a black hole of mass $10^{7} M_{\odot}$ (see Ref. ${ }^{12}$ ), one obtains that $A(\lambda)=$ 0.0157. Using now the expression for $A(\lambda)$ derived from the fit of simulated data, ${ }^{11}$ one obtains from a numerical solution of the resulting equation that $\lambda=0.548$. This value confirms that the condition for having only partial stripping of the star is satisfied. Taking an orbital period of $P=9.5 \mathrm{yr}$ (value privileged in Ref. ${ }^{20}$ ), the derived value of $\lambda$ and replacing these into eq.(1), it results that the orbital eccentricity must be about $e=0.993$. Therefore, a plausible scenario able to explain the variable X-ray episodes in IC 3599 is the following: a MS star of type A0V in a 


\section{J. A. de Freitas Pacheco}

highly eccentric orbit $(e=0.993)$ around a $10^{7} M_{\odot}$ black hole loses about $6.4 \%$ of its mass in each pericenter passage, which occurs every $9.5 \mathrm{yr}$.

Finally, it should be mentioned the dramatic event, dubbed PS1-10jh, detected in the optical and UV bands by the Pan-STARRS-1 survey ${ }^{14}$ in 2010. No associated $\mathrm{X}$-ray emission was seen and the source was identified with a non-active galaxy at $z=0.1696$. The absence of X-ray emission indicates that the temperature through the accretion disk has not attained values as high as those in the flare episodes described previously. This is probably due to a high critical Reynolds number, which imposes a low turbulence level through the accretion disk and, consequently, a small the dissipation rate of kinetic energy. Another particularity of this event concerns the lack of hydrogen lines and the presence of broad HeII emission lines, suggesting that the disrupted star was probably the He-rich core of an evolved red-giant. The light curves in different bands were reproduced with a remarkable accuracy by the hydrodynamic models developed in Ref. ,22 considering a disrupted He-core of $0.5 M_{\odot}$, a $6.3 \times 10^{6} M_{\odot}$ black hole and a critical Reynolds number $\mathcal{R}=10^{4}$, necessary to avoid temperatures exceeding $10^{6} \mathrm{~K}$ and, consequently, an important X-ray emission.

\section{Conclusions}

Supermassive black holes with masses less than $10^{8} M_{\odot}$ in a quiescent state are difficult to be detected. However, if they are responsible for a tidal disruption event, their properties can be inferred as well as those of the stellar environment in which these SMBHs are embedded. However, in order to extract usefull informations from the light curve, an accurate modeling of the event is required. Models either of the tidal disruption process or the debris evolution are still in the infancy and more detailed numerical investigations are urged.

We have shown that the recovered high X-ray emission level present in NGC 3599 eighteen months before the original detection is consistent with an unique tidal flare event originated from the disruption of a one solar mass star by a $10^{7} M_{\odot}$ black hole. Such a recovered X-ray emission corresponds to the fast raising side of the flare light curve, before maximum emission. However, new X-ray data on IC 3599 impose a revision of the model considered in Ref. ${ }^{12}$ It was shown here that a $3.2 M_{\odot}$ star in a very eccentric orbit around a $10^{7} M_{\odot}$ black hole, loses a few percent of its mass during the pericenter passage that is sufficient to explain the recurrent X-ray emission. Nevertheless these conclusions must be reinforced by more robust computations.

Up to now, about few tens of tidal disruption events candidates are known but the situation will change dramatically with the Large Synoptic Survey Telescope (LSST), which will be in operation beginning of 2023. During the survey period (about 10 years), the LSST is expected to detect about $10^{3}-10^{4}$ tidal flare candidates, which will permit in a near future detailed studies of SMBHs during their dormant state. 


\section{References}

1. B. Abbott et al., Phys.Rev.Lett. 116, 061102 (2016).

2. R. A. Remillard and J.E. McClintock, ARAA 44, 49 (2006).

3. K. Belczynski, D. E. Holz, T. Bulik, and R. O' Shaughnessy, Nature 534, 512 (2016).

4. A. M. Ghez, E. Becklin, G. Duchjne, S. Hornstein, M. Morris, S. Salim, and A. Tanner, Astron. Nachr. 324, S1 (2003).

5. A. M. Ghez, G. Duchêne, K. Matthews, S. D. Hornstein, A. Tanner, J. Larkin, M. Morris, E.E. Becklin, S. Salim, T. Kremenek, D. Thompson, B.T. Soifer, G. Neugebauer, and I. McLean I, ApJ 586, L127 (2003).

6. L. Gao, N. Yoshida, T. Abel, C. S. Frenk, A. Jenkins, and V. Springel, MNRAS 378, 449 (2007).

7. A. Soltan, MNRAS 200, 115 (1982).

8. T. A. Small and R. D. Blandford, MNRAS 259, 725 (1992).

9. S. Peirani and J. A. de Freitas Pacheco, PRD 77, 064023 (2008).

10. Ch. Filloux, F. Durier, J. A. de Freitas Pacheco, and J. Silk, IJMP D19, 1233 (2010).

11. J. Guillochon and E. Ramirez-Ruiz, ApJ 767, 25 (2013).

12. M. Montesinos Armijo and J.A. de Freitas Pacheco, ApJ 736, 126 (2011).

13. S. Komossa, H. Zhou, T. Wang et al., ApJ 678, L13 (2008).

14. S. Gezari, R. Chornock, A. Rest et al., Nature 485, 217 (2012).

15. I. Arcavi, A. Gal-Yam, M. Sullivan et al., ApJ 79, 38 (2014).

16. S. Gezari, D. Martin, B. Milliars et al., ApJ 653, L25 (2006).

17. S. Gezari, S. Basa, D. Martin et al., ApJ 676, 944 (2008).

18. S. Gezari, T. Heckman, S. Cenko et al., ApJ 698, 1367 (2009).

19. R. D. Saxton, S. E. Motta, S. Komossa and A. M. Read, MNRAS 454, 2798 (2015).

20. S. Campana, D. Mainetti, M. Colpi, G. Lodato, P. D'Avanzo, P.A. Evans and A. Moretti, $A \mathscr{E} A$ 581, A17 (2015).

21. M. Montesinos Armijo and J. A. de Freitas Pacheco, $A \mathscr{E} A$ 5 526, 146 (2011).

22. M. Montesinos Armijo and J. A. de Freitas Pacheco, MNRAS 430, L45 (2013). 